\title{
LA EMPRESA \\ PARTICIPATIVA Y LAS TIC
}

Isabel Fernández Rodríguez*

\section{RESUMEN}

RESUMEN

El artículo centra su reflexión

en la capacidad de generar

participación en la empresa, tarea en

al cual se implica la presencia de las tecnologías de información y

comunicación y se relevan algunas

herramientas específicas que

favorecen la participación en cuatro

formas: en la información y la

comunicación, en la definición de

objetivos, en la toma de decisiones y

en la creación de conocimiento.

\section{Palabras Clave}

Empresa participativa Generar participación

Tecnologías de información y comunicación
En los últimos tiempos no paramos de oír hablar de empresas más participativas como modelos de organizaciones favorecedoras de prácticas innovadoras en nuestro entorno. Tal y como señala D. Santiago García Echevarría', 'solamente cuando se disponga de instituciones y organizaciones flexibles es cuando puede innovarse. La innovación no sale de instituciones rígidas o jerarquizadas sino de instituciones empresariales con gran rapidez de adaptación y flexibilidad'.

Hablamos de organizaciones más innovadoras como factor clave para poder competir en un entorno global donde diferenciarnos de nuestros competidores, también globales, requiere de empresas que sean capaces de ofrecer bienes y servicios diferentes a los que el resto de empresas ofrecen.

Pero vayamos al grano: ¿qué entendemos por empresas participativas? Puesto que participar es algo que, en mayor o menor medida, lo hacen todas las personas de una organización, la pregunta más precisa sería ¿qué entendemos por empresas más participativas?

\footnotetext{
* Colaboradora del Cluster del Conocimiento.

1 García Echavarría, S. La revolución institucional en la empresa. Cuadernos de Empresas y Humanismo.
} 
En nuestra opinión se trata de un concepto muy ambiguo dada la diversidad de enfoques desde los que se analiza. De cara a no perdernos en debates dialécticos, nosotros defendemos una definición en su dimensión más amplia en la que asumimos que 'la participación se refiere a todos los métodos que permiten a los trabajadores influir en la gestión de la empresa'. Se trataría, por lo tanto, del grado de implicación y de compromiso que las personas de una organización adquieren en el proceso de toma de decisiones ${ }^{2}$.

Por otra parte, entendemos que una cosa es generar la capacidad de participar y otra muy diferente es generar el deseo de participar por parte de las personas. En este artículo vamos en centrarnos precisamente en el primer aspecto: generar la capacidad de participar.

Para ello, tomamos como base de partida las formas de participación que definen Ayestarán, S. et al [2006] en su publicación 'Rumbo a la innovación: Trabajo en equipo y cambio cultural en las organizaciones'. En su investigación, distinguen cuatro formas de participación:

- Participación en la información y comunicación, forma en la que se exige transparencia de la información y acceso a la misma de las personas que conforman la organización.

- Participación en la definición de objetivos, donde la aportación de opiniones por parte de las personas así como el conocimiento individual y grupal de la metodología PDCA [Planificar, Hacer, Evaluar y Ajustar] resultan aspectos clave para que la participación se pueda dar.

2 Cuesta, I., y Fernández, I. «Modelo Vasco de Empresas Participativas». (2001).

\section{Abstract}

This article makes a reflection on the capacity to generate company participation, a task which implies the presence of information and communication technologies, and some specific tools

which favor participation in three different ways are also described, taking into account information and communication, the definition of clear aims, decision making and creation of knowledge.

\section{KEY WORDS}

Participative company Generating participation Information and Communication technologies. 
- Participación en la toma de decisiones, individual y/o colectivamente, donde necesariamente las personas deben conocer el despliegue de el/los proceso/s en los que toman parte, de cara a la toma de decisión más idónea.

- Participación en la creación de conocimiento, donde el mero hecho del intercambio de información no es suficiente: ha de darse además una coordinación, una integración de ideas, perspectivas y experiencias que faciliten el descubrimiento de una nueva idea.

Retomando la frase de D. Santiago García Echevarría con la que comenzamos esta reflexión 'La innovación no sale de instituciones rígidas o jerarquizadas sino de instituciones empresariales con gran rapidez de adaptación y flexibilidad' nos percatamos de que las TIC [Tecnologías de la Información y Comunicación] juegan un papel fundamental. La intensidad con que se ha dado la llamada revolución de las TIC ha producido un impacto directo en las formas de organizarse, han permitido que el mundo pueda no continuar siendo un lugar compuesto de compartimentos estancos sino un lugar donde las conexiones entre individuos y organizaciones sean más posibles, más ágiles y más flexibles; en definitiva, las organizaciones planas son organizaciones que favorecen la actuación en red y esto, es posible gracias a las TIC.

Y a raíz de estas reflexiones nos preguntamos, ¿tenemos hoy en día a nuestra disposición herramientas TIC que ayuden a generar capacidad de participar en la gestión de las organizaciones de una manera flexible? Nosotros pensamos que sí. Hoy en día contamos con herramientas que nos permiten fomentar la participación en la información y comunicación, en la definición de objetivos [estratégicos y tácticos], en la toma de decisiones y en la creación de conocimiento, siendo esta última desde nuestro punto de vista, el gran reto de nuestra sociedad.

A continuación, señalamos una serie de soluciones [destacamos sólo algunas de ellas] que, desde nuestro punto de vista, usadas correctamente, pueden favorecer las cuatro formas de participación que hemos mencionado:

- Weblog o blog: según definición de wikipedia, 'es un sitio web periódicamente actualizado que recopila cronológicamente textos o artículos de uno o varios autores, apareciendo primero el más reciente, donde el autor conserva siempre la libertad de dejar publicado lo que crea pertinente. Mediante un formulario se permite, a otros usuarios de la web, añadir comentarios a cada entrada, pudiéndose generar un debate alrededor de sus contenidos'. Aplicado a empresas, puede fomentar la gestión de conocimiento de 'abajo a arriba', facilita la coordinación de equipos dispersos geográficamente y fortalece la cultura interna de la empresa.

- ERP [Enterprise Resource Planning]: se trata de un conjunto de aplicaciones que facilitan la gestión y la integración de los distintos procesos que intervienen en la cadena de valor de la empresa. Proveen de herramientas que permiten compartir información entre los procesos y comunicarse entre ellos, de manera que las personas que componen la empresa disponen de información precisa para la toma de decisiones, en todos los niveles.

- KM [Knowledge management]: herramienta que proporciona sistemas que facilitan la gestión documental de la empresa así como su clasificación y búsqueda de manera rápida y sencilla. 
Asimismo, puede tener otras funcionalidades como son foros virtuales que permiten compartir experiencias entre las personas que conforman la organización.

- Business Intelligence [Inteligencia empresarial o de negocio]: según definición de wikipedia, 'conjunto de estrategias y herramientas enfocadas a la administración y creación de conocimiento mediante el análisis de datos existentes en una organización o empresa. Este conjunto de herramientas y metodologías tienen en común las siguientes características: accesibilidad a la información, apoyo en la toma de decisiones y orientación al usuario final. Se trata por tanto, del proceso de análisis de datos de la empresa para poder extraer conocimiento de ellos. Con BI se puede: crear una base de datos de clientes, prever ventas y devoluciones, compartir información entre diferentes departamentos, mejorar el servicio al cliente'.

Sin embargo queremos destacar que el reto no sólo consiste en identificar las herramientas más adecuadas para el tipo de participación que queremos fomentar en nuestra organización. Es necesario pero no suficiente: resulta clave conocer el impacto que esa herramienta va a provocar en las formas de trabajo de las personas que componen la organización. ¡Cuántas empresas han realizado inversiones en herramientas que no se están aprovechando adecuadamente!

Y tú, ¿conoces lo que las herramientas TIC pueden hacer por tu organización para conseguir una mayor participación de las personas que la componen? ¿Conoces las implicaciones que puede traer consigo su implantación, el impacto en las formas de trabajo y en las personas? 